\title{
Language Use in Social Media and Natural Language
}

\author{
Omoregbe, Esohe Mercy \\ Professor of Linguistics \\ Department of Linguistics Studies \\ University of Benin \\ Benin City, Nigeria \\ Idada, Osasogie Christa \\ Post Graduate Student(Masters) \\ Department of Linguistics Studies \\ University of Benin \\ Benin City, Nigeria
}

\begin{abstract}
This research is a comparative analysis of language used in social media and natural language. Due to the increase in technological innovations over the years, the different social media platforms have adopted different language styles as a means of communication. The trend of this language style has led to different varieties of language such as acronyms, abbreviations, symbols, etc. which tend to differ from the natural discourse. This research set out to compare the pattern of communication in social media and natural language, as well as show how abbreviations, Acronyms and symbols are used in social media discourse to replace sentential constructions in natural language discourse. The differences that exist between these two language styles was alsodiscussed. The relevance of this research is to examine its linguistic characteristics and its implication to the field of linguistics as well as the cause of the variation of language among internet users which now pose a threat to natural language. The research concludes that communication on social media has shaped our face to face interaction and modes of communication are linguistically different from the other.
\end{abstract}

Keywords: Social Media, Natural Language, language styles, Comparative Linguistic,

\subsection{Introduction}

Social media depends onweb-based technology to create highly interactive platforms through which individuals and communities share, co-relate, discuss and modify user generated content (Kaplan and Haenlein, 2010:61). On the other hand, Natural language as used here, refer to face-to-face interaction or communication between individuals or group without any form of computer mediation. Face-to-face interaction is the reciprocal influence of individual upon one another's actions when in one another's immediate physical presence. Natural languages use speech as a means of communication. It is the ability of persons to process sentences in a natural discourse rather than on a specialized artificial computerized platform. The comparison between language used in social media and natural language is that done between online interaction and face-to-face interaction.Since the beginning of the $21^{\text {st }}$ century, the advent of different social sites or social networks has been on the increase. The coming of these different social platforms is to serve the purpose of social interaction or online communication. This has also led to the development of different language styles adopted by people on social media which is quite different from natural language. This research, a comparative analysis of the language use in social media and natural language analyse the differences and similarities that exist between social media language use and natural (face-to-face) language use.The aim of this research is to carry out a comparative analysis of language use in social media and natural language discourse. The set objectives include to:

1. compare the pattern of communication in social media discourse and natural language discourse;

2. show how abbreviations, acronyms and symbols are accommodated in social media discourse to replace sentential constructions in natural language discourse; and

3. to discuss the similarities and differences that exist between language use in social media discourse and natural language discourse. 


\subsection{Methodology}

The data for this research were gotten from both primary and secondary sources. The primary data includes oral and written data. The oral data collection was done through recording and observation of natural language using writing materials and a voice recorder. The written data on the other hand were gotten by extracting excerpts of digital communication from chat rooms (such as Facebook, Whatsapp, Twitter, Telegram, etc.), short message system (SMS), and Emails. The sources of the natural language use data were friends, course mates, family members, church settings and the students' hostel. The informants for the social media language use are friends, family members, course mates and students residing in the hostel including neighbours who gave us permission to look through their chats messages and relevant exchange of information made online.

The research was also supported by information from textbooks; online and hard print, and journal articles. The informants are located in Benin and are between the ages of 12 to 30 years. The relevant data selected from the bulk of data collected were then subjected to verification by linguists, bloggers and active social media users. This was done so as to account for the integrity of the data. After the authentication of the data, the researcher then subjected the data to a systematic comparative discussion and analysis.The study is delimited to comparative analysis that is similarities and differences between social media language and natural language.

\subsection{Previous Studies}

A report conducted by Robinson and Stubberud (2012:105) confirms that university students and other members of the next generation are highly involved with using technology to communicate and stay connected with friends. According to them "keeping in touch regardless of location could be said to enhance one's social presence, which is the perception of awareness of communication partner or the degree to which a person is perceived as real in mediated communication". The report also went further to show that slightly over $60 \%$ of students prefer to communicate using short message system (SMS) texting, almost $40 \%$ use the telephone, almost 30\% e-mail, and about $10 \%$ prefer social networking, instant messaging or face-to-face methods. The statistics above are evidence that communicating with technology is now becoming the central means of sending messages rather than face-to-face meetings. A research conducted by Subramanian, K.R. (2017:76) on the influence of social media in interpersonal communication examined the impact of social media in the way people communicate with each other. The research findings are that social media has changed the way in which people communicate-the face to face interaction has reduced. Also, the impact of social media is more advantageous than its disadvantages. In his conclusion, nothing can replace face to face interaction that is; it remains the best form of social interaction.

\section{Theoretical Framework}

\subsection{Dell Hymes- Ethnography of Communication}

Hymes 1964 proposed the ethnography of communication as an approach towards analysing patterns of language used within the speech communities. In order to provide support for his idea of communicative competence which itself was a reaction to Noam Chomsky's distinction between linguistic competence and linguistic performance. Hymes originally coined "ethnography of speaking" in his 1962 eponymous paper but was redefined in his 1964 paper, "Introduction: Toward ethnography of communication" to accommodate for the non-vocal and non-verbal characteristics of communication. Although most ethnography of communication researchers still tend to focus on "S.P.E.A.K.I.N.G" as it is generally considered to be a prominent-even primordial means of communication. The term ethnography of communication is meant to be descriptive of the characteristics that an approach towards language from an anthropological standpoint must take. According to Hymes;

1. it must investigate directly the use of language in context situation so as to design patterns proper to speech activity and;

2. to take as context a community investigating its communicative habits as a whole.

In other words, rather than divorcing linguistics from its function, the analysis of a culture's or community's communication, linguistic and otherwise, must occur with respect to the socio-cultural context of its use and the function of meanings conveyed. Hymes (1974:9) states that, "the starting point is the ethnographic analysis of the communicative conduct of a community" communicative conduct is what people do when they communicate with one another. He set out to show that a researcher could use his methods to study this communication (talk) systematically. Hymes offered a mnemonic device of the S.P.E.A.K.I.N.G grid as heuristic. The eight components of the S.P.E.A.K.I.N.G mnemonic are;

1. S- The first letter ("S") designates situation, which includes both the scene and setting. This is where the activities are taking place and the overall scene in which they are apart. 
2. P- The second refers to the participants involved. This area includes people present and the roles they play or the relationships they have with other participants.

3. E- The ends or goals of communication to be studied.

4. A- Acts or speech acts include both form and content that is any action can be considered communicative action if it conveys meaning of participants.

5. K- The key or tone of speech. How the speech sounds were delivered.

6. I- Instrumentality or the channel through which communication flows can be examined.

7. N- Norms of communication or the rules guiding talk and its interpretation can reveal meaning.

8. G- Finally Genres, one can look at cultural or traditional speech genres such as proverbs, apologies etc.

\subsection{Comparative Linguistics}

This is also another approach to be adopted in carrying out this research. Comparative linguistics is the differences and similarities that exist among languages. The primary reason for comparative linguistics is the explanatory interest of gaining a better understanding of the casual process involved in the production of an event, feature or relationship. The two conventional types of comparative linguistics focus on the explanation of differences and the explanation of similarities. The reason is that what counts as a similarity or differences depends not only on the observed values but also on the analyst and should therefore be regarded as a social construct rather than as an objective reality.In conclusion, the relevance of these two theoretical frameworks in carrying out this research is based on the fact that the study involves a comparison that is the language use in social media and language use in natural language and in analysing these different language styles ethnography of communication as propounded by Dell Hymes in 1964 will be employed to account for the different language use, styles and patterns using the S.P.E.A.K.I.N.G mnemonic. Dell Hymes ethnography of communication is a theoretical framework while comparative linguistics that is the differences and similarities that exist between the language use on social media and natural language is a descriptive approach. Thus, the present research will involve both theoretical and descriptive analyses.

\subsection{Data Analysis and Discussion}

The data collected for this research include different forms of language use adopted on social media which is further classified using a table under different sub-headings such as those with the retainment of consonants, retainment of vowels, replacement of letters with numbers and acronyms. The language use on social media is mostly characterized by abbreviations, symbols and acronyms which are used to replace sentential constructions in natural (face-to-face) language discourses.

Table 1: The Retainment of Consonants

\begin{tabular}{|l|c|c|}
\hline \multicolumn{3}{|c|}{ Retainment of Consonants } \\
\hline a) & Bt & But \\
\hline b) & Bc & Because \\
\hline c) & Nvr & Never \\
\hline d) & Brk & Freak \\
\hline e) & Fwd & Work \\
\hline f) & Wrk & Friend \\
\hline g) & Frnd & Not \\
\hline h) & Nt & People \\
\hline i) & Pls & Serious \\
\hline j) & Srs & Next \\
\hline k) & Nxt & Text \\
\hline 1) & Txt & Thanks \\
\hline m) & Trx & Heart \\
\hline n) & Hrt & Senior \\
\hline o) & Snr & \\
\hline
\end{tabular}

In the retainment of consonants, vowels are usually deleted while only the consonants are retained. Guy (1991) writes, "Prior studies are unanimous that a following consonant promotes deletion more readily than a following vowel". Consonants usually at the initial, middle and final positions of words are retained especially when a vowel is a preceding letter after the initial consonant. In the above table the retainment of consonant has been observed to result to consonant clusters. Consonant cluster is the occurrence of more than one consonant together. Therefore consonants when retained can either form an abbreviated word such as 'Next'-Nxt, 'Forward'-Fwd, 'Work'-Wrk, 'Senior'-Snr, 'But'-bt, 'Heart'-Hrt, or an acronym such as 'Laughing Like Shit'-LLS, 'GirlFriend'-GF, 'Happy Married Life'-HML, 
'YouTube'-YT, 'Best Regards'-BR, 'Laughing But Very Serious'-LBVS, 'Know How You Feel'-KHYF, 'No Big Deal'-NBD. This shows that most abbreviations or acronyms when retained have effect on the words that is later formed (consonant cluster) thereby neglecting the importance of vowels in words formation which was originally to break words and prevent consonant sounds from clustering.

Table 2: The Retainment of Vowels

\begin{tabular}{|l|l|l|}
\hline \multicolumn{3}{|l|}{ Retainment of Vowels } \\
\hline a) & You & U \\
\hline b) & Hey & A \\
\hline c) & Hi & I \\
\hline d) & Yours & Urz \\
\hline e) & I Agree & IA \\
\hline f) & I owe you & Iou \\
\hline g) & User interface & Ui \\
\hline h) & Eye & I \\
\hline
\end{tabular}

In the retainment of vowels, consonants are usually deleted while only the vowels are retained. Some vowels when retained can either be replaced with a single vowel as in the data the word 'you'-U, 'Hi'-i, 'Hey'-A was replaced with a single vowel while others maybe as a result of abbreviations or acronyms such as 'I Agree'-IA, 'I Owe You'-IOU, 'User Interface'-UI.

Table 3: The Replacement of Letters with Numbers

\begin{tabular}{|l|l|l|}
\hline \multicolumn{3}{|c|}{ Replacement of letters with numbers } \\
\hline a) & 29 t & To night \\
\hline b) & B4n & Bye for now \\
\hline c) & Ate & 8 \\
\hline d) & 143 & I love you \\
\hline e) & 2 L8 & Too late \\
\hline f) & 4 & For \\
\hline g) & B4 & Before \\
\hline h) & L8 & Late \\
\hline i) & $<3$ & Heart or love \\
\hline j) & 9 t & Night \\
\hline k) & n & Foreign \\
\hline l) & F9 & Fine \\
\hline m) & GRL & For real \\
\hline n) & G9 & Genius \\
\hline o) & 2 & Too, to \\
\hline p) & Ba3 & Battery \\
\hline
\end{tabular}

Replacement of letters with numbers is one of the prominent features of electronic communication. The example above shows us clearly that the number options are limited, that is from 1-9. The most frequently used figure is ' 2 ' for the infinitival marker 'to' or the adverb 'too' and number '4' for the proposition 'for'. One other option which may be found in electronic language is the figure ' 8 ' typically found in the word great-gr8. Also in the substitution of letters for numbers the whole word may be deleted and substituted for numbers as in 'to' or 'too'-2, 'for'-4, 'ate'-8, 'one'-1, 'I Love You'-143, 'heart'-3. This shows a complete replacement of letters with numbers while there is also the partial replacement of letters with numbers. These letters are usually the combination of letters with numbers or numbers blending with letters such as, 'Bye for now'-b4n, 'Genuis'-G9 with, 'foreign'-4N, 'too late'-2L8, 'battery'-Ba3.

Table 4: The use of Acronyms

\begin{tabular}{|l|l|l|}
\hline \multicolumn{2}{|l|}{ The use of acronyms } \\
\hline a) & LOL & Laughing out loud \\
\hline b) & OMG & Oh my Gosh/god \\
\hline c) & LMAO & Laughing my ass out \\
\hline d) & IMO & In my opinion \\
\hline e) & PITA & Pain in the Ass \\
\hline f) & ROFL & Rolling on the floor laughing \\
\hline
\end{tabular}




\begin{tabular}{|l|l|l|}
\hline g) & BRB & Be right Back \\
\hline h) & SMH & Shaking my head \\
\hline i) & HBD & Happy Birthday \\
\hline j) & TY & Thank you \\
\hline k) & WB & Welcome back \\
\hline
\end{tabular}

Acronyms are usually expressions of phrasal sentences reduced into single words and this is as a result of the need to be concise in online communication. Acronyms are formed using the first letter of each words that makes up the phrasal sentence while other letters after the initial letter (that is, whether consonants or vowels) are deleted. According to Tagliamonte and Denis (2008) in studying the patterns online language also found only two prominent acronyms in the entire corpus which are Laughing Out Loud-LOL and Oh My Gosh/god-OMG. Acronyms can either be a combination of both consonants and vowels as in 'Laughing My Ass Out'-LMAO, 'In My Opinion'-IMO, 'Pain In The Ass'-PITA, 'Rolling On The Floor Laughing'-ROFL. Also acronyms could be as a result of retained consonants such as 'Be Right Back'-BRB, 'Shaking My Head'-SMH, 'Happy Birthday'-HBD, 'Thank You'-TY, 'Welcome Back'-WB or as a result of retained vowels, 'I Owe You'- IOU, 'User Interface'-UI, 'I Agree'-IA, 'Integration Order'-IO. Furthermore, acronyms can also be a combination of numbers and letters such as 'Got To Go'-G2G, 'Face To Face'F2F, 'Got TO Tell You'-G2TU, 'Back To Business'-B2B, 'Explain Like I'M 5(years old)-ELI5.

\subsection{Features of the Language Use on Social Media}

The data collected shows the features of language style adopted by most social media users as a means of communication. These features include:

\section{The Use of Abbreviations}

Abbreviation is from the Latin word 'brevis' meaning short. It is a shortened form of a word or phrase. An abbreviation may be made by omitting certain portions from the interior or by cutting off a part. Widespread use of electronic communication through mobile phones and the internet during the 1990s allowed for a marked rise in colonial abbreviation. This was due largely to increasing popularity of textual communication services such as instant and text messaging. Short message service (sms), for instance, supports message lengths of 160 characters at most. This brevity gave rise to an informal abbreviation scheme sometimes called textese, with which $10 \%$ or more of the words in a typical short message service (SMS) message are abbreviated. More recently, twitter, a popular social networking service began driving abbreviation use with 140 character message limits. Examples of some abbreviations commonly used on social media are shown in the table below:

Table 5 shows abbreviations used in social media conversation

\begin{tabular}{|ll|l|}
\hline (i) & afak & as far as I know \\
\hline (ii) & afk & away from keyboard \\
\hline (iii) & asap & as soon as possible \\
\hline (iv) & atw & at the weekend \\
\hline (v) & bbl & be back later \\
\hline (vi) & bcnu & be seeing you up \\
\hline (vii) & brb & be right back \\
\hline (viii) & btw & Between \\
\hline (ix) & cu & see you \\
\hline (x) & cm & call me \\
\hline
\end{tabular}

These abbreviations are used most frequently in chat groups and virtual worlds, though they have spread to e-mail, web pages and even to non-internet print forms and sometimes into speech in everyday life.

\section{Acronyms}

An acronym is an abbreviation formed from the initial components in a phrase or a word. Usually these components are individual letters or parts of words or names. There are broad currents of consensus but no universal standardization of various names for such abbreviations. Whereas an abbreviation maybe any type of shortened form, such as words with the middle omitted (for example Dr. for Doctor), an acronym is a word formed from the first letter or first few letters of each word in a phrase. 
Table 6: Some acronyms commonly used in social media

\begin{tabular}{|l|l|l|}
\hline (i) & CTA & Call to Action \\
\hline (ii) & $\mathrm{G}^{+}$ & Google plus \\
\hline (iii) & FB & Facebook \\
\hline (iv) & HBD & Happy Birthday \\
\hline (v) & HML & Happy Married Life \\
\hline (vi) & LI & Linkined In \\
\hline (vii) & ESP & Email Service Provider \\
\hline (viii) & IG & Instagram \\
\hline (ix) & PV & PageViews \\
\hline (x) & S M & Social Media \\
\hline (xi) & SERP & Search Engine Results Page \\
\hline (xii) & DM & Direct Message \\
\hline (xiii) & SM P & Social Media Platform \\
\hline (xiv) & SOV & Share of Voice \\
\hline
\end{tabular}

The above acronyms are mostly used by social media users on different social media platforms as a means of communication.

\section{Symbols}

A symbol is an object that represents, stands for, or suggests an idea, visual image, etc. Symbols take the form of words, sounds, gestures or usual images and are used to convey ideas and beliefs. Symbols are a means of complex communication that often can have multiple levels of meaning. Social media users, use emoji. They are picture characters designed to show emotional facial expressions.

\subsection{Differences between language used in social media and natural language}

Crystal (2001:29-40) drew several major differences between the language used on social media which he referred to as "Netspeak" and face-to-face conversations. According to him, lack of simultaneous feedback is a major distinction between the language used on social media and face-to-face conversations. In netspeak messages sent via computer are complete and unidirectional. When we send a message to someone, we type it a keystroke at a time but it does not arrive on that person's screen a keystroke at a time-in the manner of the old tele-printers. The message does not leave our computer until 'we send it', and that means the whole of a message is transmitted once. There is no way that a recipient can react to our to our message while it is been typed, for the obvious reason that recipients do not know they are getting any messages at all until the text arrives on their screens. The second difference between netspeak and faceto-face conversation also results from the technology: the rhythm of an internet interaction is very much slower than that found in speech situation and disallows some of conversations' most salient properties. With e-mails and asynchronous chat groups, a response to stimulus may take anything from seconds to minute, the rhythm of the exchange of information is very much depending on such factors as the recipient's computer (example, whether it announces the instant arrival of a message), the user's personality and habits (example, whether messages are replied to at randomly), and the circumstances of the interlocutors (example, their computer access).

The time delay (usually referred to as lag) is a central factor in many situations. There is an inherent uncertainty in knowing the length of the gap between the time of posting a message and the moment of receiving a reaction. Because of lag, the rhythm of interaction - even in the fastest netspeak encounters, in synchronous chat groups and virtual worlds lack the pace and predictability of that found in telephonic or face-to-face conversation. Even if a participants types a reply immediately, there may be delay before that message reaches the other members screen due to several factors such as bandwidth processing problems, traffic density on the host computer, or some problem in the sender's or receiver's equipment.Netspeak lack facial expression, gestures and conventions of body posture and distance (the kinetics and proxemics) which are so critical in expressing personal opinions and attitudes and in moderating social relationships. The limitation was noted in the early development of netspeak, and led to the introduction of smiley or emoticons. According to Crystal and Quirk (1964) emoticons have been called "the paralanguage of the internet", and their absence does not mean that the user lacks emotion conveyed. In face-to-face communication, someone may grin over several utterances and the effects maybe noted. In netspeak, a 'grin' emoticon might be added to just one utterance, although the speaker may continue to 'feel' the relevant emotion over several turns, there is also no guarantee that the person who sends a 'grin' is actually grinning at all- a point which also applies to abbreviations used: how many people are actually "laughing out loud" when they send LOL?. The use of smiley as a mechanism devised to get round the absence of kinetic and proxemics features but on the whole netspeak lacks any true ability to signal meaning through kinetic and proxemics features. 
Furthermore, one objective of using these technologies is to create comparable levels of communication speed and effectiveness as those achieved in traditional face-to-face meetings. According to Hollenbeck et al., (2008:793) states that the important difference between traditional face-to-face communication and computer-mediated communication is that the former encompasses more psychological connections" due to the impact of social presence cues in virtual communication settings.

He also went further to states that "during computer- mediated communication, the level of social presence has decreased leading to a less effective communication experience". But Hatem et al., (2011:30) argued that, "performance of technology mediated communication is higher than that of face-to-face contact because the lack of social presence in the media communication requires less individual and socio-emotional interaction, thus resulting to amore task oriented form of collaboration". Mehra (2012:30) goes on to say that, "lack of various non-verbal and social context cues to collaborate in computer-mediated technology may hamper the effectiveness of task performance".

Computer-mediated communication system has imposed a constraint on communication that is likely to affect a group's performance. People rely on multiple modes of communication in face-to-face conversation such as Para verbal (tone of voice, inflection, voice volume) and non-verbal (eye movement, facial expression, hand gestures and other body language) cues.

These cues help to regulate the flow of conversation, facilitate turn-taking, provide feedback and convey subtle meanings. As a result face-to-face conversation is remarkably an orderly process. Hatem et al., (2012:383) stated that, "the participants in face-to-face communication tend to be consciously aware of the non-verbal messages which are being sent to each other but nevertheless, they respond to these signals which can be very powerful", such communication modalities are constrained to a varying extent depending on the characteristics of the technological system. For example, electronic mail (e-mail) prevents both Para verbal and non-verbal cues, telephone conference calls allows the use of most Para verbal cues ( but not non-verbal ones) while video conferencing (skype) enables extensive use of both Para verbal and non-verbal cues. kirkman, (2012:57) stated that "the absence of social interaction and non-verbal cues may even create an important level of anonymity and thus leads to a higher incidence of rude and offensive behaviour in computer-mediated technology in contrast to face-to-face contact".

Also virtual teams are not able to duplicate the normal "give and take" of face-to-face discussion. For example comments of group members using a synchronous computer-mediated communication system sometimes appear to be out of context, or the conversation may appear to lack focus because multiple group members are "talking" at once. Group members who type slowly or edit more thoroughly may find their comments are no longer relevant when they are ready to transmit them. Moreover, because everyone can transmit their comments simultaneously, group members may be required to process a large number of comments in a short period of time. For a synchronous computermediated communication system, considerable delays typically occur between the time a message is sent and the time a reply is received. This may make it difficult to maintain a train of thought or a discussion theme.

\subsubsection{The Impact of language used in social media on natural language discourse}

Recent technological advancement has had a drastic impact on the way individuals communicate. Little by little, technology has become an integral part of the way that people communicate with one another and is gradually taking the place of face-to-face communication. The drastic increase in technology usage is especially noticeable in younger generations. One study conducted by the Kaiser family foundation, found people ages 8 to 18 spent more time on social media than any other activity at an average of 7.5 hours a day (Foehret al., 2010). Many studies have been conducted regarding technology's effect on social interaction and face-to-face communication since the rise of cellphone and social media usage in the late 2000s. According to Przybylski and Weinstein (2012:1) "Recent advancement in communication technology has enabled billions of people to connect more easily with people great distances away, yet little has been known about how the frequent presence of these devices in social setting influences face-to-face interactions".

Also Przybylski and Weinstein (2012) showed similar results that proved the presence of mobile communication devices in social settings interferes with human relationships in two separate experiments by the authors found evidence that these devices have negative effects on closeness, connection and conversation quality, especially when individuals are engaging in personally meaningful topics. Brignall and Van Valey (2005:337) analyzed the effects of technology among "current cyber-youth" - those who have grown up with the internet as an important part of their everyday life and interaction rituals. The two authors discovered that due to the pervasive use of the internet in education, communication and entertainment, there has been a significant decrease in the amount of time spent on faceto-face interaction among youth. They suggest that the decrease in the amount of time youths spent interacting face-toface may eventually have "significant consequences for their development of social skills and their presentation of self."As development of online communicative language might be different from one country to another, Ross (2006) believed that apart from borrowing some features from English, some of the features in the online communicative 
language are actually home-grown, and display their own identity and cultural values. Language evolution is seen as an on-going process, and the development of online communicative language is always unpredictable even though it might originate from a re-think process of some old spelling conventions in the media, and not some patterns of language that derived from its own. Jehnova (2004) found that online communicative features only appeared at $20 \%$ of the entire conversations $13 \%$ of the utterances containing sole acronyms.

The research found 7.2\% (279) of 'LOL' (Laugh Out Loud) as the highest number of acronym been used in the chat rooms. The other acronyms would include 263 acronyms of 'PMI' (Please Message Later); 37 'FFS,' (for f**k sake); 23 'TY' (Thank You); 16 'LMAO' (Laugh My Ass Off); 14 'WB' (Welcome Back); 14 'OMG' (Oh My God); 13 WTF (What the F**k); 12 'ASL' (Age, Sex, Location); 9 'BRB' (Be Right Back) and 7 'PC' (Personal Computer).As the evolution of online communicative language is moving along with time or even speed faster than the time, there are still on-going debates on the "acceptance" of online communicative language among sociolinguists. Scholars express different concerns from different perspectives; some are in favour of its existence and some show approval but a bit conscious on the long term effects it might bring on students' proficiency skills. Ross (2006) suggests several factors that contribute to the emergence of many short forms in online communicative language such as the need to be fast in responding to others.

"Speedy communication allows less time for careful, organized thought, partly for this reason, e-mails, text messages and broadcast messages (instant messages, chat messages) are in many ways stylistically more similar to spoken language than traditional written forms." (Ross, 2006:41)

It is assumed that online communication settings might also serve as a new platform that allows users to ignore the need to be accurate in spelling which could be an advantage for those who are actually having problems in spelling words accurately. Crystal (2006) perceived that online communication is actually a platform that gives its users a chance to practice the language with no fear of display, mistakes and a huge freedom to be creative with the language. This is supported by Baron (2008:169) who believes that the current situation indicates a beginning of a new set of language rules and cultures as people are adapting themselves with a more "casual attitude towards linguistic consistency."

\section{Conclusion}

Many forms of new media technologies such as computer, cellphones, smart phones, web camera chat, texting, social networks, blogging, android tablets, Ipad's have taken a new hold on the paradigm of face-to-face communication. More and more people of all ages are using technology to communicate for a variety of purposes including business, formal and informal situations. There are clear signs of the emergence of a distinctive variety of language with characteristics closely related to the properties of its technological context as well as intentions, activities and personalities of users. Many new technologies are anticipated which will integrate the internet with other communication situations and these will provide the matrix within which further online language varieties will develop. As people decide to use social media to communicate more and talk to people less, a potential decline of verbal and non-verbal skills, social skills, writing skills and lack of human presence may occur in face-to-face interaction.

From the research conducted on the comparative analysis of language used in social media and natural language shows the evolving of a new media language on social media which has been found to be different from natural language. The features of the language use in social media show a wide usage of acronyms, abbreviations and symbols instead of sentential constructions when chatting. These features of social media language have resulted in springing up different language varieties which are posing threats to the writing, verbal and non-verbal language skills of individuals. Language being such a sensitive index of social change, it would be surprising if such a radically innovative phenomenon do not have a corresponding impact on the way we communicate. Language use in social media will doubtlessly grow in its socio-Linguistics and stylistic complexity to be comparable with already known traditional faceto-face interaction. The development of internet language poses difficulty in predicting its existence because of so many conflicting trends and pressures. The Net is immensely empowering individualistic creative medium as can be seen from the numerous experimental ways in which people use it.

\subsection{Recommendation}

The electronic evolution which has brought about a linguistic revolution in social media language and natural language has become part of a much larger computer-mediated language which in the 'digitally designed' form enhanced the community's linguistic norm. Whereas, at the moment, face-to-face communication ranks as primary in any account of the linguistic potentialities of human kind but in the future, it may not be so. In statistical sense, we may one day communicate with one another far more through computer mediation than in face-to-face interaction. Therefore, areas not covered by this research such as the use of emojis in computer mediated communication should be point of study as it is fast substituting words on social media platforms. 


\section{References}

Baron, S.L. (2008). Always On: Language in an Online and Mobile World. New York: Oxford University Press.

Brignall, T.W. and Van Valley, T. (2005). The Impact of Internet Communication on Social Interaction. Sociological Spectrum. $335-348$.

Crystal, D. (2001). Language and the Internet. Cambridge: Cambridge University Press.

Crystal, D. (2006). Language and the Internet. ( $2^{\text {nd }}$ Ed.). Cambridge: Cambridge University press.

Crystal, D. and Quirk, R. (1964). Systems of Prosodic and Paralinguistic Features in English. The Hague: Mouton.

Foeher, U.G., Rideout, V.J. and Roberts, D.F. (2010). Generation MZ: Media in the lives of 8-to-18 year old. Kaiser Family Foundation. 1 - 85.

Guy, G.R. (1991). Contextual Conditioning in Variable Lexical Phonology. Language variation and change. 3, 223 239.

Hatem, W., Kwan, A., and Miles, J. (2011). Comparison of Face-to-Face a Computer Mediation Collaboration. Http://ctwbisq/i.ctw.utwente.n// hartmannt/proceedings/papers\%20in\%20pdf/2.pdf.

Hatem, W., Kwan, A., and Miles, J. (2012). Comparing the Effectiveness of Face-To-Face and Computer-Mediated Collaboration. Advanced Engineering Informatics. 26(2), 383 -395.

Hollenbeck, C.R., Song, J.H. and Zinkhan, G.M. (2008). The Value of Human Warmth: Social Presence Cues And Computer Mediated Communication System.Advanced in consumer research. 357, 93 - 794.

Hymes, D.H. (Ed.). (1964).Language in Culture and Society: A reader in linguistics and anthropology. New York: Harper \& Row.

Hymes, D.H. (1974). Foundation in Socio-Linguistics: An Ethnographic Approach. Philadelphia: University of Pennsylvania press.

Jehnova, M. (2004). The language of chat. An online Journal of Modern Philology.ISSN1214-5505. Retrieved 25 December, 2009 from http://philologial.net/studia/2004013000003.

Kirkman, B., Triana, M. and Wagstaff, M. (2012). Does the Order of Face-to-Face and Computer-Mediated Communication Matters in Diverse Project Teams? An Investigation of Communication Order Effects on Minority Inclusion and Participation.Journal of Business and Psychology. 27(1), 57-70.

Kplan, A.M. and Haelein, M. (2010). Users of the World, Unite! The Challenges and Opportunities of Social Media. Business Horizon. 53(1), 61.

Mehra, P. (2012). Task Interactions and The New Media: Does More Frequent Interaction Contribute To Greater Satisfaction With The Media? Singapore management Review. 34(2), 30-48.

Pryzybylski, A.K; and Winstein, N. (2012). Can you Connect Me Now? How the Presence of Mobile Communication Technology Influences Face-to-Face Conversation Quality.Journal of Social and Personal Relationship. 1 10.

Robinson, S. and Stubberud, H. (2012). Communication Preferences among University Students.Academy of Educational Leadership Journal. 16 (2), 105-113.

Ross, N. (2006). Writing in the Information Age. English Today. 22 (3), 41 - 113.

Subramanian, K.R. (2017). Influence of Social Media In Interpersonal Communication. International Journal of Scientific Progress and Research. Issue 109, 38(2) 70-75

Taglimonte, S.A. and Denis, D. (2008). Linguistic Ruin? Lol? Instant Messaging and Teen language. American Speech.83, 3 -34. 